\title{
ULTRASOUND APPLICATION IN PHYSICAL THERAPY OF DOGS: COMPARATIVE STUDY
}

\author{
Verebová, ${ }^{1}{ }^{1}$, Staničová, J. ${ }^{1,2}$ \\ ${ }^{1}$ Department of Chemistry, Biochemistry and Biophysics \\ University of Veterinary Medicine and Pharmacy in Košice, Komenského 73, 04181 Košice \\ Slovakia \\ ${ }^{2}$ Institute of Biophysics and Informatics, First Medical Faculty \\ Charles University, Salmovská 1, 12000 Prague 2 \\ Czechia
}

valeria.verebova@uvlf.sk

\begin{abstract}
The application of ultrasonic methods in veterinary medicine, especially in the therapy of dogs, is the main objective of this study. We compared the frequency of therapeutic ultrasound using in rehabilitation as well as in microsurgical interventions of dogs in Slovakian and Hungarian veterinary practices. Regarding to the evaluation of survey realized in restricted regions, the ultrasound therapies and interventions are currently used in Slovakia less than in Hungary. Our study could start a change in this unfavourable aspect in Slovakian veterinary medicine and contribute to a better promotion of ultrasound application in the therapy of animals.
\end{abstract}

Key words: dogs; questionnaire; rehabilitation; ultrasound therapy

\section{INTRODUCTION}

Mechanical waving field includes the waves in a broad frequency range namely from $2 \mathrm{~Hz}$ to $200 \mathrm{MHz}$. The range is divided in relation to human audibility into three approximate intervals: infrasound, audible or acoustic sound, and ultrasound. The ultrasound (frequencies above $20 \mathrm{kHz}$ ) is an essential tool in medicine in both diagnostic as well as therapeutic applications. Recently, it has become obvious that no human or veterinary clinic and hospitals exist without a sonography which is a widespread diagnostic method based on ultrasound waves. Short ultrasound waves are spreading directly through the environment, which enables an application of their reflection on tissue boundaries as the basic phenomenon in sonography [13].

Diagnostic information is obtained by capturing, processing, and displaying of ultrasound signals reflected from tissue interfaces. These signals are subsequently received by an ultrasonic probe [3]. The information about the investigated tissues, their depths, and possible artefacts found within the body is given by evaluation of reflected ultrasound waves [1].

Nowadays, the ultrasound field has a broad application in human and veterinary medicine. Besides using sonography for diagnostics, there are a lot of applications in therapy and surgery. Figure 1 presents a brief comprehension of ultrasound utilization in medicine. Therapeutic effects 


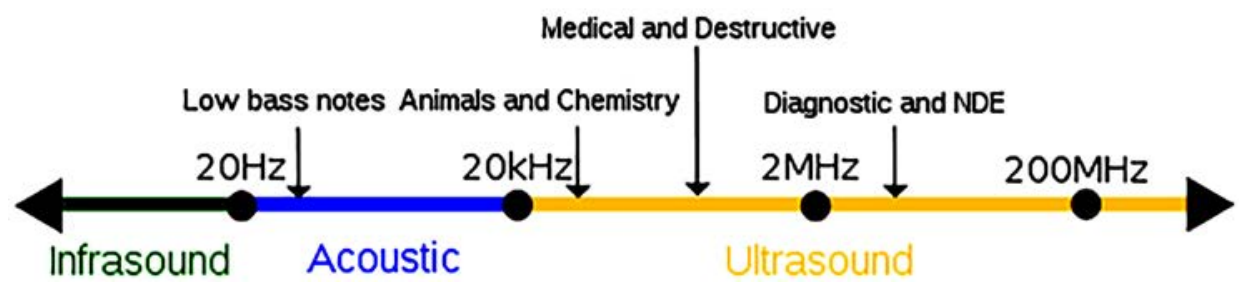

Fig. 1. Spectrum of mechanical waves NDE-non-destructive effects

of ultrasound can be divided into destructive effects and non-destructive effects (Fig. 1).

Ultrasound frequencies above $100 \mathrm{kHz}$ and below $2 \mathrm{MHz}$ have destructive effects; they can be used in many medical applications. For example, Extracorporeal Shockwave Lithotripsy (ESWL) is a sophisticated method using for fragmentation and removing of urinary, bile or kidney stones. Other prospective methods such as releasing of drugs from their delivery systems (liposomes, microspheres) or High Intensity Focused Ultrasound (HIFU) which is used for the ablation of tumours are based on the utilization of frequencies from the above mentioned range. Phacoemulsification in ophthalmology and cleaning of teeth in dental hygiene are methods based on the destructive effects of ultrasound as well. Recently they apply predominantly in human medicine with the exception of tartar removing, which is a common method in veterinary dental medicine.

In general, ultrasound evokes primary and secondary effects when it interacts with biological organisms. The majority of ultrasound effects are presented by the transformation of mechanical energy to thermal or chemical energy within tissues. Non-thermal effects are called cavitation: vibration of tissues causes a formation of microscopic bubbles - they stimulate cell membranes and enhance the cell-repair effects of the inflammatory response. It is clear that these effects are utilized predominantly in physiotherapeutic procedures in physical therapy or rehabilitations [3].

Therapeutic ultrasound is a physiotherapy technique that aims to accelerate the healing of damaged muscles, tendons or ligaments by reducing inflammation. Physical therapists have made use of therapeutic ultrasound for the therapy of human patients since the 1940's, and veterinarians have used it on animals since the 1970's. It is applied in various fields of veterinary medicine such as surgery, dentistry, inhalation therapy, or physiotherapy. In medical practice, therapy by ultrasound has many benefits such as non-invasive intervention in the patient's body leading to faster recoalescence, increasing blood flow in the treated area and thereby accelerating the treatment process, alleviation of oedemas and heat production in the tissues that help in reducing pain and muscle spasms [4].

In our study, we would like to point out the possibility of ultrasound application in the treatment of dogs. We summarized and compiled the current state of ultrasound utilization as therapeutic method in Slovakia (from the perspective of dog owners and veterinarians) and compared it with the trend abroad (specifically in Hungary).

\section{Application of ultrasound in physical therapy}

The purpose of physical therapy (physiotherapy, rehabilitation) is to accelerate the healing of damaged muscles, joints, tendons, and ligaments by reducing their inflammation. Excellent results have been obtained in acute injuries as well as in chronic lesions [14]. The rehabilitation effect of ultrasound is caused by the absorption of ultrasound waves by individual molecules and macromolecules within the tissue.

When the tissue contains a lot of protein macromolecules, after the ultrasound, the absorption given by absorption coefficient is higher and vice versa: the absorption coefficient decreases when water content within tissues increases. This fact has various beneficial effects including increased blood flow (which promotes healing), reducing swelling, and acceleration the comprehensive healing process. Penetration depth which is the maximum depth when a rehabilitation effect is expected, has reached in the frequency interval $0.9-3 \mathrm{MHz}$ with few W. $\mathrm{cm}^{-2}$ of ultrasound intensity $[9,11]$. Currently, there is not enough published scientific clinical trials focused on ultrasound physiotherapy and the used exposures (doses) are largely empirically determined. Most physiotherapy devices of- 
fer a probe emitting ultrasound intensities in the range of $0.2-3 \mathrm{~W} . \mathrm{cm}^{-2}$ and discrete frequencies $1-3 \mathrm{MHz}$ for deep treatment (to depth of $8 \mathrm{~cm}$ ) and $3 \mathrm{MHz}$ for surficial treatment. Ultrasound physical devices allow either discrete intensity (pulsed regime) settings or continuously (continuous regime) variable control.

Setting of the work cycle parameters applied to the patient depends on diagnosis and the fact whether the problem is acute or chronic. Usually, the acute diseases need to adjust lower intensities because of the excessive heating of the tissue (only $60 \%$ of the intensity, which is necessary for the treatment of chronic diseases) $[6,10]$.

Physiotherapy by using the ultrasound is a very simple procedure which must be repeated several times ( 2 to 3 weekly sessions with 10-20 minute applications are needed depending on the patient's condition) for achievement of a satisfied therapeutic effect. The total duration of the treatment depends on the particular patient and diagnosis $[4,12]$. The ultrasound produced by special therapeutic probes is coupled directly into the patient through a thin layer of coupling medium (e.g. aqueous gel or mineral oil) [5]. It is necessary to keep the ultrasound probe in the continuous contact with the skin during the therapy. Insufficient contact absorbs a large amount of energy resulting in a lower intensity of ultrasound wave motion in the tissues. The therapeutic unit works in so-called scan mode, which represents the longitudinal movement of the probe along the muscle $[4,14]$.

Each treatment form has also its undesirable effects. The unfavourable effects of ultrasound physical therapy include a status worsening of inappropriate dosing (intensity, duration of application), overall weakness, short term temperature rise, insomnia, vomiting, and extension at the application side [3]. The ultrasound rehabilitation is not recommended for contraindications such as an orthopaedic implant or any metallic components in the patient's body (overheating and subsequent damage to the surrounding tissue may occur). It also is not to be used in patients with cancer, infected areas of the skin over the foetus during pregnancy, growth platelets in young animals, open spine after surgery, eyes or over uncastrated male testicles [4].

\section{Application of ultrasound in surgery, micro-interventions and dental medicine}

Surgical therapy has developed more slowly in view of its technical difficulties and possible larger risks for the patient. The ultrasound with intensity up to $20 \mathrm{~W} . \mathrm{cm}^{2}$ and frequency from interval $100 \mathrm{kHz}-2 \mathrm{MHz}$ (Fig. 1) is used to destroy pathological tissues that could also damage the surrounding healthy tissue or organs when it is applied incorrectly [3]. The ultrasonic aspirator is the most popular ultrasound instrument used in general surgery. It belongs to the contact surgical instruments. Oncologists most often use it for removing the primary tumours and metastases of the liver, kidney, breast, bone, uterus, and pancreas. The advantage of this method is that ultrasound quite hardly disturbs rigid and elastic tissues with high collagen content (blood vessels, bronchi, and various ligaments). Surrounding soft tissues are disturbed by ultrasound and then can be easily aspirated away, thereby tissues with higher levels of collagen are exposing. The ultrasonic aspirator also has a thermo-coagulation effect which reduces the bleeding from small blood vessels $[7,8]$.

Dental tartar is one of the most commonly diagnosed dental problems in animals, especially in dogs. Ultrasonic units used in dentistry are currently available in two basic types; their mechanism of action is different. Magnetostrictive units operate between 18 and $45 \mathrm{kHz}$ using flat metal strips or metal rod attached to a scaling tip and the tip movement is elliptical. Piezoelectric units operate in the $2-50 \mathrm{kHz}$ range and are reactive by dimensional changes in the crystals housed within the hand piece and the tip movement is primarily linear in direction [2].

\section{MATERIALS AND METHODS}

To monitor the current state of using the ultrasound therapy in veterinary practice (especially in the therapy of dogs), we have chosen two forms of questionnaire. The first form was addressed to the dog owners in Slovakia and it was published on the internet. The second one was assigned to veterinarians, it was sent via e-mail, and the doctors were contacted by phone. We also monitored the application of ultrasound therapy abroad, specifically in Hungary, by sending questionnaires to Hungarian veterinarians. We do not know the total number of dog owners contacted as it is not possible to know how many saw the questionnaire on the internet but did not reply to us. The number of veterinarians to whom we either sent the questionnaires by e-mail or contacted by telephone was 209 (153 from Slovakia and 56 from Hungary). 


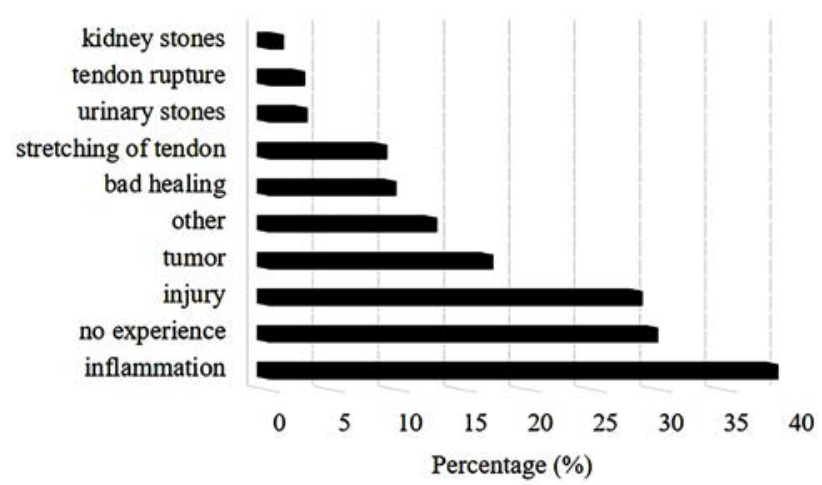

Fig. 2. Experiences of dog owners with individual types of diseases

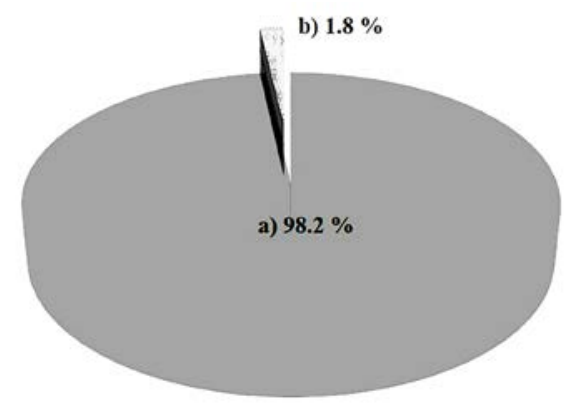

Fig. 4. Real experience of dog owners with ultrasound therapy; a) no experience or the owners confused ultrasound therapy with diagnostics, b) positive experience

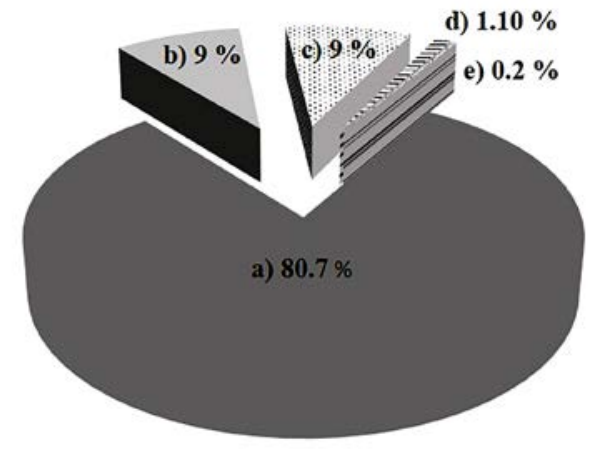

Fig. 3. The possibility of ultrasound rehabilitation offered by a veterinarian; a) no, b) I don't remember,

c) the therapy is not available, d) no injury of the dog, e) yes

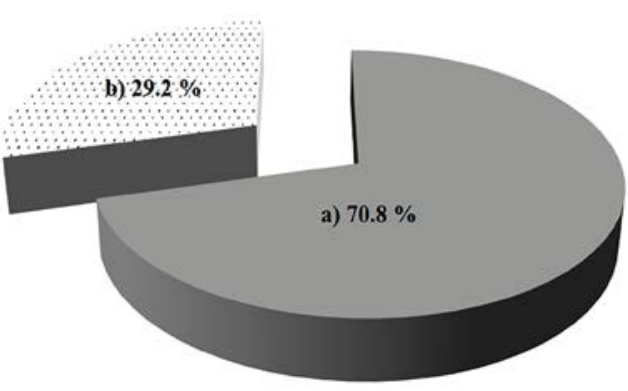

Fig. 5. Awareness of dog owners about ultrasound rehabilitation; a) no, b) yes

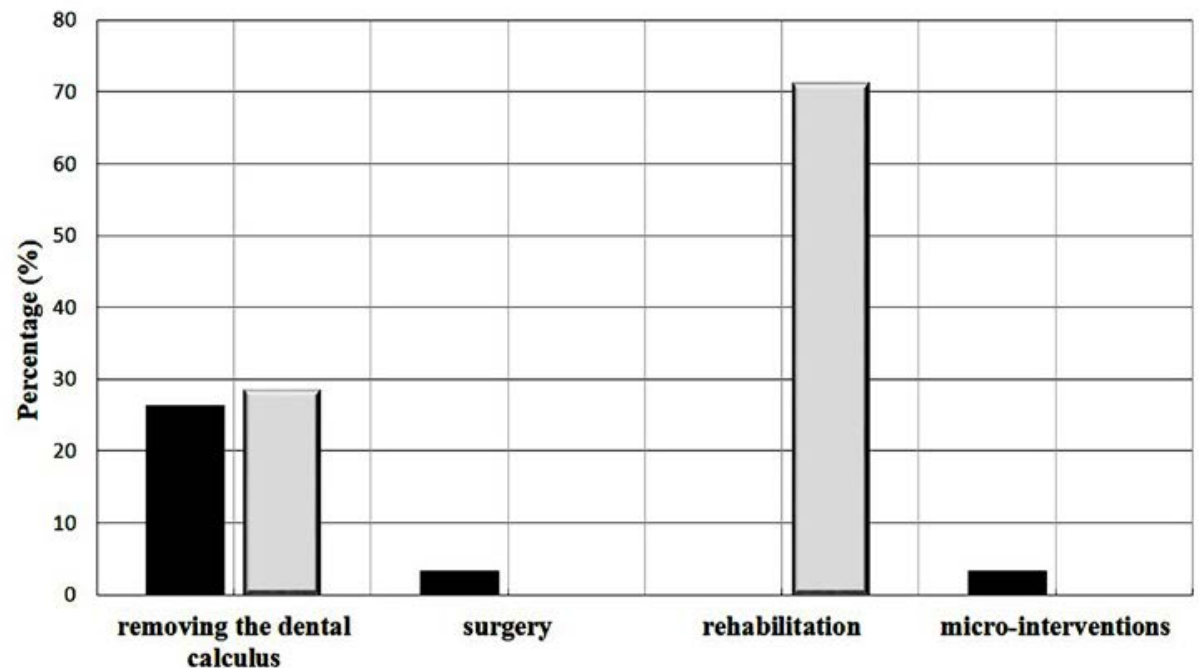

Fig. 6. Comparison of ultrasound therapy applications in Slovakia and Hungary; Slovakia-black, Hungary-grey 


\section{Questionnaire for dog owners}

The questionnaire for dog owners consisted of nine questions. It contained three structured questions (the owner had the choice of several answers), three open questions (the respondent was allowed to answer individually), and the three semi-closed questions (the possibility to choose an answer or answer individually). General information such as their age or sex were considered in the questionnaire. The questionnaire was preferentially focused on the owners' experiences with ultrasound therapy.

\section{Questionnaire for veterinarians}

The questionnaire addressed to the veterinarians was designed by a veterinarian was more concise and contained two open and two closed questions. All of questions that were proposed have led to the collection of information about veterinarians' knowledge, use, and experiences with ultrasound therapy applications on dogs' patients.

\section{RESULTS}

\section{Evaluation of the questionnaire done by dog owners}

Four hundred and forty-five dog owners coming from Slovakia were included in the study. The average age of the respondents was 32 years, of which 55 were men and 390 were women. The age of their dogs ranged from 2 months to 17 years with the average age being 6 years.

In question number six, we asked the dog owners if they had experience with any of these types of diseases (injury, tendon strain, urinary and kidney stones, tumour, tendon rupture, inflammation or poor healing). Up to $38.9 \%$ of dog owners had experience with inflammation, $28.5 \%$ with injury, $17.1 \%$ with tumour, $9.7 \%$ with poor healing, $9 \%$ with tendon extension, $2.9 \%$ with urinary stones, $2.7 \%$ with tendon rupture and $1.1 \%$ with kidney stones. Almost a third of the owners (29.7\%) answered that they have no experience with any disease of their dog. $12.8 \%$ of respondents mentioned the possibility of "another" (they did not specify the type of disease) (Fig. 2).

In another question, we were interested in whether dog owners were offered the possibility of ultrasound rehabilitation by veterinarians, if the patient's condition required rehabilitation. $80.7 \%$ of respondents were not offered such a possibility by a veterinarian, $9 \%$ stated that they did not remember it, and $1.1 \%$ of owners answered that their dog did not have an injury, did not need such a form of therapy. Only a very low percentage $(0.2 \%)$ answered that they were offered the option of ultrasound rehabilitation (Fig. 3). Even today, there are surgeries that do not have the therapeutic ultrasonography.

The eighth question of the questionnaire focused on the personal experiences of dog owners with ultrasound therapy, specifically with ultrasound rehabilitation. However, the majority of responses (98.9\%) were negative, as respondents had no experience with this type of therapy. Only $1.1 \%$ of owners had the positive experience with ultrasound rehabilitation.

In processing the answers to this question, we found that a very large percentage of dog owners (Fig. 4) confused ultrasound therapy with ultrasound diagnostics. Respondents most often stated that they encountered ultrasound when diagnosing diseases or detecting pregnancy in their bitch.

"Have you ever heard about ultrasound form of rehabilitation?", this was the ninth question of our questionnaire. $70.8 \%$ of respondents confirmed that they have not heard about this method yet and $29.2 \%$ of interviewees knew about this method (Fig. 5).

\section{Evaluation of the questionnaire done by veterinarians}

Thirty veterinarians from Slovakia and seven veterinarians from Hungary responded to the questionnaire intended for them. For comparison, we present together the results of a questionnaire obtained from both countries, Slovakia and Hungary.

In the first question, we were interested in whether veterinarians have and use an ultrasonograph. Of the randomly selected outpatient clinics throughout Slovakia, they have and utilize $100 \%$ diagnostic ultrasound. The situation is the same in Hungary, all surgeries we contacted are equipped with the ultrasonography and use it.

In the next question, we focused on medical procedures in which veterinarians use ultrasound. Approximately onethird (32.9\%) of the surveyed veterinarians in Slovakia apply ultrasound in addition to diagnostics but also for the removal of the tartar $(26.7 \%)$, in surgery (30.3\%) and in micro-interventions (30.3\%) (Fig. 6). Hungarians use ultrasound for therapy much more, namely, $71.4 \%$ for rehabilitation and $28.6 \%$ for tartar removal in dog patients.

From Fig. 6, it can be seen that Hungarian veterinarians utilize ultrasound as an effective tool for rehabilitation of 
dogs; with the difference to Slovak vets, they do not use ultrasound for rehabilitation at all. Almost $72 \%$ of Hungarian vets used ultrasound rehabilitation on the therapy of arthrosis, neurological problems, lymph stimulation, metabolism acceleration, tendinitis, bursitis, and joint diseases.

\section{DISCUSSION}

Ultrasound therapy is one of the newer therapeutic methods, so we were interested in the knowledge and experiences associated with application of this therapy in dogs, their owners, and the veterinarian in their communities. Although our study does not depict a large sample of veterinarians and dog owners, the results are more than surprising. From the above-mentioned questionnaire, it is clear that among the most common dogs' difficulties in which their owners look for professional veterinary help include inflammation, injury, and cancer. Less often, there occurs kidney and urinary stones, stretching or rupture of tendons. Up to $80 \%$ of owners mentioned that if rehabilitation was needed, they were not offered the opportunity to apply ultrasound therapy. As can be seen from Fig. 6, the Hungarian veterinarians utilize ultrasound as an effective tool for dog rehabilitation more frequently than Slovak veterinarians. Almost $72 \%$ of Hungarian vets use ultrasound rehabilitation on the therapy of arthrosis, neurological problems, lymph stimulation, metabolism acceleration, tendinitis, bursitis, and joint diseases. Slovak vets are predominantly concerned with micro interventions by ultrasound, such as removing tartar and micro surgery.

When we have processed and evaluated the results, we also found that approximately $98 \%$ of dog owners exchange ultrasound therapy with sonography. This phenomenon is especially widespread in Slovakia. We suppose that it is related to the low promotion of application, benefits, efficiency and effectiveness of ultrasound therapy, and especially rehabilitation in the lay public. We were pleasantly surprised by the fact that the lay public as demonstrated in the questionnaire has the ambition to obtain more information and knowledge about possibilities of using ultrasound therapy in health problems to alleviate and improve the welfare of their pets. It may be advisable to or- ganize a series of popular lectures designed for laypersons/ dog owners focusing on the benefits and effectiveness of ultrasound therapy. This would certainly help to boost the awareness of veterinarians contributing to increased interest and enhanced use of effective non-invasive method such as ultrasound therapy. Increased interest in this form of therapy by owners could assist veterinarians overcome barriers (higher financial contributions, profitability) that are likely to prevent them from using ultrasound therapy and rehabilitation to a greater extent.

\section{CONCLUSIONS}

Innovative therapeutic methods in veterinary medicine include ultrasound therapy. Rehabilitation by ultrasound leads to faster recovery, accelerates the healing process, reduces oedema, pain and occurrence of muscle spasms. In this study, we wanted to provide a consistent overview on the ultrasound application in dog therapy; specifically rehabilitation in Slovakia. By using a questionnaire, we have compiled the current state of using ultrasound therapy in practice. We compared the results with the situation in Hungary. It is clear from our study that Slovakia lags behind the current trend, which dominates abroad. Most veterinarians use ultrasound only for diagnostics as assistive technique in surgery, in catheterization or elimination dental tartars in dog patients. No participating doctors use ultrasound for rehabilitation treatments. The trend prevailing abroad, especially in Hungary, shows a tendency for an increased number of ultrasound applications in arthrosis, rehabilitation, tendon injuries, dampening pain and inflammation, and oedema reduction.

Finally, we can conclude that ultrasound therapy is currently very little used in Slovakia, which is obstructive to its positive aspects. Our study could contribute to a better promotion of this expanding rehabilitation method.

\section{ACKNOWLEDGEMENTS}

Authors would like to thank to Katarina Bačová for helping with the dissemination of questionnaires. 


\section{REFERENCES}

1. Adámek, D., Cihlář, F., Hořejší, L., 2010: Advances in ultrasonography in the diagnosis of renal tumours. Urol. Pract., $11,257-261$

2. Arabaci, T., Cicek, Y., Canakci, C. F., 2007: Sonic and ultrasonic scalers in periodontal treatment: A review. Int. J. Dent. Hyg., 5, 2-12. DOI: 10.1111/j.1601-5037.2007.00217.x.

3. Čech, E., 1982: Ultrasound in Medical Diagnostics and Therapy. Avicentrum, Prague, 432 pp.

4. Downing, R., 2012: Ultrasound Therapy. https://vcahospitals. com/know-your-pet/therapeutic-ultrasound. Accessed on April 6th, 2021

5. Haar, G., 1999: Therapeutic ultrasound. Eur. J. Ultrasound, 9 , 3-9. DOI: 10.1016/S0929-8266(99)00013-0.

6. Henoch, Q., 2014: Ultrasound Machine Setup. https://www. youtube.com/watch?v=QfyGqQ5QOHE. Accessed on May 7th, 2021

7. Choi, J. S., 2012: The SAGES Manual on the Fundamental Use of Surgical Energy (FUSE). Springer, New York, USA, 133-138.
8. Kennedy, J. E., Haar, G., Cranston, D., 2003: High intensity focused ultrasound: surgery of the future. Br. J. Radiol., 76, 590-599. DOI: 10.1259/bjr/17150274.

9. Poděbradský, J., Vařeka, I., 1998: Physical Therapy. Grada, Prague, CR, $440 \mathrm{pp}$.

10. Pye, S., Milford, C., 1992: The performance of ultrasound therapy machines in Lothian region. Ultrasound Med. Biol., 20, 347-359. DOI: 10.1016/0301-5629(94)90003-5.

11. Rosina, J., 2000: Medical Biophysics. Manus, Prague, CR, $524 \mathrm{pp}$.

12. Sathish, R., 2018: Ultrasound Massage and Sonic Wave Therapy for Jasper. https://www.youtube.com/watch?v=mOZ4kJyvA5U. Accessed on April 17th, 2021.

13. Szabo, T. L., 2004: Ultrasound Diagnostic Imaging: Inside Out. Elsevier Academic, Boston, USA, 549 pp.

14. Wernham, B. G. J., Jarram, R., Warman, C. G. A., 2008: Bicipital tenosynovitis in dogs. Compendium, 30, 537-552.

Received September 23, 2021

Accepted October 25, 2021 